\title{
Vehicle Platoons Through Ring Coupling
}

\author{
Jonathan A. Rogge and Dirk Aeyels
}

\begin{abstract}
In this paper, a novel strategy for the control of a string of vehicles is designed. The vehicles are coupled in a unidirectional ring at the interaction level: each vehicle is influenced by the position of its immediate forward neighbor; the first vehicle in the platoon is influenced by the position of the last vehicle. Through these interactions a cooperative behavior emerges and a platoon of vehicles moving at a constant velocity with constant inter-vehicle spacings is formed. This contrasts with more traditional control schemes where an independent leader vehicle is followed by the remaining vehicles.

For this control structure, stability properties are established. The concept of string stability of a platoon is discussed and applied to the ring interconnection. Design rules are presented, showing how an appropriate choice of parameter values leads to a constant spacing or constant time headway policy. Furthermore, the scheme has a characteristic property: it maintains the platoon structure when subject to malfunctioning vehicles.
\end{abstract}

Index Terms-Ring coupling, string stability, vehicle platoons.

\section{INTRODUCTION}

I $\mathrm{N}$ this paper the systems under study are vehicular platoons. Such systems have gained importance over the years, as they help relieving the congestion of highways in urban areas. The objective being a capacity increase of the highway, these intelligent vehicle/highway systems (IVHS) form strings of vehicles (platoons) moving at a desired speed with desired distances between the vehicles. Several algorithms controlling a string of vehicles have been proposed in the literature.

[1]-[3] rank among the first to investigate this problem by using an LQR approach. Recently, this approach has been readdressed in [4]. Contrary to [1] and [2], most control strategies use tuning of parameters in order to optimize a particular controller. In most cases the control is of leader-follower type: the leading vehicle of the platoon moves at a desired speed; the other vehicles receive information from the leading vehicle (position, velocity, acceleration) either directly or indirectly through other vehicles in the platoon. Flow of information is usually directed from the head of the platoon towards its tail [5], [6]. In [7] for instance, a control strategy is developed where each vehicle adjusts its behavior according to the leading vehicle and its forward neighbors.

An important concept regarding the formation of vehicle platoons is string stability. A platoon is called string stable if the transient error in the inter-vehicle spacing does not grow as one proceeds down the line of vehicles. In [8], [9] the concept of

Manuscript received May 15, 2006; revised July 6, 2007.Published August 29,2008 (projected). This work was supported in part by the Belgian Programme on Interuniversity Attraction Poles, initiated by the Belgian Federal Science Policy Office. Recommended by Associate Editor G. J. Pappas.

The authors are with the SYSTeMS Research Group, Department of Electrical Energy, Systems and Automation, Ghent University, Zwijnaarde B9052, Belgium (e-mail: jonathan.rogge@ugent.be; dirk.aeyels@ugent.be).

Digital Object Identifier 10.1109/TAC.2008.925812 string stability is discussed for an infinite number of vehicles. In Section IV adaptations of the definition in [8] are introduced in order to accommodate the systems investigated in this paper, consisting of a finite number of vehicles.

The present paper introduces a novel interconnection topology using identical controllers. The key property of the interconnection is the absence of a master/leader vehicle, which determines the overall behavior of the platoon. Instead, each vehicle interacts with other vehicles, trying to satisfy its individual control objective. As a result of this cooperation a platoon formation emerges: the vehicles move at a constant velocity with constant distances between each pair of consecutive vehicles. The paper is organized as follows. The behavior of the vehicle string and its stability properties are discussed in Sections II and III. String stability of the system is established in Section IV. A robustness property characteristic for the ring coupling scheme is demonstrated in Section V, illustrating the tendency of the platoon not to break when subject to malfunctioning vehicles.

\section{SYSTEM DYNAMICS}

\section{A. Ring Topology}

The idea of ring coupled agents has been exploited before in [10]-[13]. In [10] each agent $i$ is represented by a point $z_{i}$ in the complex plane and the dynamics are

$$
\dot{z}_{i}=\left(z_{i+1}+c_{i}\right)-z_{i}, \quad i \in \mathcal{I}:=\{1, \ldots, N\}
$$

where $c_{i} \in \mathbb{C}$, and $z_{N+1}:=z_{1}$. If $\forall i \in \mathcal{I}, c_{i}=0$, then the agents will converge to one point in the complex plane. Choosing values $c_{i}$ appropriately leads to desired formations of the group of agents. If the centroid of the points $c_{1}, \ldots, c_{N}$ is not at the origin, then the centroid of the agents moves off to infinity. This situation is undesired in the setting of [10].

In [11] the topology of the interconnection network is a unidirectional ring, as in [10] and the present paper, but the dynamics of each individual agent is different. Each agent is represented as a kinematic unicycle with nonlinear dynamics. The control strategy is such that agent $i$ tries to reduce its distance to agent $i+1$ to zero. This is done by a proportional feedback of the difference in heading, i.e., orientation, of both vehicles

$$
\left[\begin{array}{c}
\dot{x}_{i} \\
\dot{y}_{i} \\
\dot{\theta}_{i}
\end{array}\right]=\left[\begin{array}{cc}
\cos \theta_{i} & 0 \\
\sin \theta_{i} & 0 \\
0 & 1
\end{array}\right]\left[\begin{array}{c}
s \\
k\left(\theta_{i+1}-\theta_{i}\right)
\end{array}\right]
$$

where $s$ and $k$ are positive real constants. The resulting equilibrium motion has all agents moving along a circle in one direction. The motion in the physical plane clearly reflects the unidirectional interconnection structure.

In the present paper, the vehicles are coupled in a unidirectional ring at the level of interaction, similar to [10] and [11]. 
However, the ring topology is not expressed at the level of the geometry of the formation: the platoon consists of a string of vehicles moving on a line. As will be made explicit in the following sections, assigning values to parameters in the system determines the velocity and inter-vehicle spacings of the platoon.

To conclude this section, we present a lemma on circulant matrices, adopted from [14], which introduces notation used in the next section, and which is applied in the stability analysis in Section III.

Lemma 1: Consider the block circulant matrix $C \in$ $\mathbb{R}^{N m \times N m}$

$$
C=\left[\begin{array}{cccc}
C_{1} & C_{2} & \cdots & C_{N} \\
C_{N} & C_{1} & \cdots & C_{N-1} \\
\vdots & \vdots & \vdots & \vdots \\
C_{2} & C_{3} & \cdots & C_{1}
\end{array}\right]=: \operatorname{circ}\left(C_{1}, C_{2}, \ldots, C_{N}\right)
$$

where $C_{i} \in \mathbb{R}^{m \times m}$, for all $i$ in $\mathcal{I}$. The matrix $C$ can be block diagonalized into a matrix $\Lambda$

$$
\Lambda=\operatorname{diag}\left(\Lambda_{1}, \ldots, \Lambda_{N}\right)
$$

where the blocks $\Lambda_{i} \in \mathbb{C}^{m \times m}$ are given by

$$
\Lambda_{i}=C_{1}+\omega^{i-1} C_{2}+\omega^{2(i-1)} C_{3}+\cdots+\omega^{(N-1)(i-1)} C_{N}
$$

for all $i$ in $\mathcal{I}$, with $\omega:=\exp (2 \pi j / N)$, where ' $j$ ' represents the imaginary unit.

\section{B. System Equations and Platoon Solutions}

Each vehicle $i \in \mathcal{I}$ is represented as a moving point mass satisfying

$$
\ddot{x}_{i}+p \dot{x}_{i}=u_{i}
$$

where $x_{i}$ represents the position of the $i$ th vehicle, $u_{i}$ is the input to the $i$ th vehicle and $p \geq 0$ is a parameter representing the friction/drag coefficient per unit mass [1]. The mass of each vehicle is taken equal to one.

Consider the input

$$
u_{i}=\omega_{i}, \forall i \in \mathcal{I}
$$

where $\omega_{i} \geq 0$. In an ideal situation, setting all inputs $u_{i}, i \in \mathcal{I}$, equal to a constant $\omega>0$, the vehicles' velocities converge to the same value with inter-vehicle spacings depending on the initial conditions. The rate of convergence is determined by the drag coefficient and cannot be tuned. In a practical situation the values $\omega_{i}$ differ from each other, leading to unwanted behavior. To remedy the situation, the input of each vehicle is modified

$$
u_{i}=\omega_{i}+K\left(x_{i-1}-x_{i}-L_{i}-h \dot{x}_{i}\right), \quad i \in \mathcal{I}
$$

with $K>0$ the coupling strength, and $h>0, L_{i}, i \in \mathcal{I}$, real constants. The ring interconnection is made explicit by defining $x_{0}:=x_{N}$. Equations (4) and (6) yield the dynamical system

$$
\dot{x}=A x-K b+\omega
$$

where

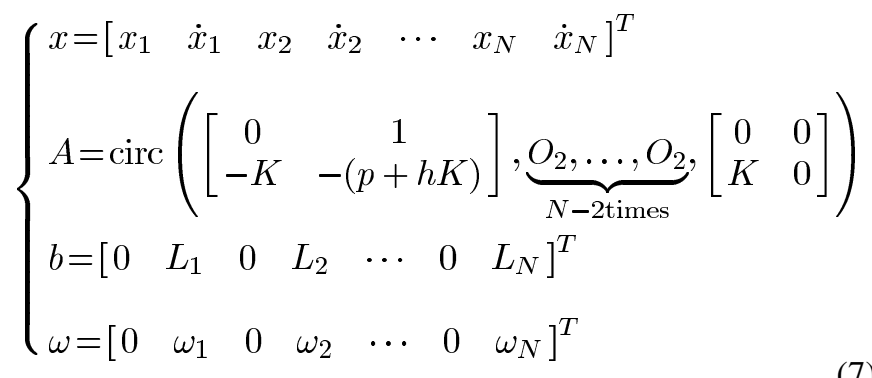

with $O_{2}$ the $2 \times 2$ null matrix. Notice that initial conditions should be chosen such that they respect the order of the vehicles and such that collisions are avoided at the start. The behavior of the system is described in the following theorem.

Theorem 1: The function $\varphi: \mathbb{R} \rightarrow \mathbb{R}^{N}$ defined by

$$
t \mapsto \varphi_{i}(t)=\alpha t+\beta_{i}, \quad \forall i \in \mathcal{I}
$$

where

$$
\begin{aligned}
\alpha & =\frac{\omega_{m}-K L_{m}}{p+h K} \\
\beta_{i}-\beta_{i-1} & =\frac{\omega_{i}-\omega_{m}}{K}+L_{m}-L_{i}
\end{aligned}
$$

with $\omega_{m}:=1 / N \sum_{j=1}^{N} \omega_{j}, L_{m}:=1 / N \sum_{j=1}^{N} L_{j}$, and $\beta_{0}:=$ $\beta_{N}$, is a solution of system (7).

Proof: We show that the given values of $\alpha$ and $\beta_{i}-\beta_{i-1}$, $\forall i \in \mathcal{I}$ follow from the substitution of (8) into (7). This substitution yields

$$
\alpha p=\omega_{i}-K \beta_{i}+K \beta_{i-1}-K L_{i}-\alpha h K, \quad \forall i \in \mathcal{I} .
$$

Equation (8) represents a solution of (7), if and only if $\alpha, \beta_{i}$ satisfy (10). Adding all $N$ equations (10) leads to a value for $\alpha$

$$
\alpha=\frac{1}{N(p+h K)} \sum_{j=1}^{N}\left(\omega_{j}-K L_{j}\right) .
$$

Equation (10) can be written as

$$
\beta_{i-1}-\beta_{i}=\frac{\alpha(p+h K)}{K}+L_{i}-\frac{\omega_{i}}{K}, \quad i \in \mathcal{I} .
$$

With (11), this changes into

$$
\beta_{i-1}-\beta_{i}=\left(\frac{1}{N} \sum_{j=1}^{N} \frac{\omega_{j}}{K}-\frac{\omega_{i}}{K}\right)-\left(\frac{1}{N} \sum_{j=1}^{N} L_{j}-L_{i}\right)
$$

for all $i \in \mathcal{I}$.

A solution $\varphi$ defined by (8)-(9) is called a platoon solution of the system. In Section III it is proven that, under appropriate conditions, every solution of (7) converges to a platoon solution.

Remark: The values $\beta_{i}$ are defined up to a constant, according to (9b). If $\varphi$ is a platoon solution, then so is $\varphi+\nu \mathbb{1}$, $\forall \nu \in \mathbb{R}$, where $\mathbb{1} \in \mathbb{R}^{N} ; \mathbb{1}_{j}:=1, \forall j \in \mathcal{I}$. All platoon solutions only differ in absolute position of the platoon. We refer to them as the platoon solution. The dynamics are thus invariant 
under translations of the origin in the (one-dimensional) physical space. This invariance is reflected in the spectrum of the system matrix $A$ of system (7): the matrix $A$ possesses at least one zero-eigenvalue, corresponding to the eigenvector $\left[\begin{array}{lllllll}1 & 0 & 1 & 0 & \cdots & 1 & 0\end{array}\right]^{T}$, independent of the parameter values. For more information on spatially invariant system theory, the reader is referred to [15].

\section{Selecting Design Parameters}

In this section the longitudinal control of a vehicle string is discussed. Longitudinal control consists of speed control, regulating the platoon velocity, and spacing control, adjusting the inter-vehicle spacings. Two particular, well-known policies in spacing control are the constant spacing policy (CSP) and the constant time headway policy (CTHP). In CSP, the inter-vehicle spacings are kept constant, irrespective of the velocity of the platoon. In CTHP, the desired inter-vehicle spacings are proportional to the speed of the vehicles. This control policy takes into account that a faster vehicle requires a longer braking distance.

First, consider the longitudinal control of a leader-follower interconnection. The leader vehicle is considered as an external input to the system; it is allowed to change its velocity freely. This vehicle is responsible for the speed control of the platoon. A speeding up or slowing down maneuver of the leader vehicle results in speeding up or slowing down the entire platoon. The spacing control is implemented by giving each follower vehicle an input of the form (6) with $\omega_{i}=0$. The follower vehicles regulate the spacing with their forward neighbor according to either CSP $(h=0)$ or CTHP $(h \neq 0)$.

The situation in the present paper is significantly different. In system (7) there is no external control of the platoon. However, each vehicle has a control, described by (6), which is determined by the parameter values of $L_{i}, \omega_{i}$, and $h$ (the value of $K$ is considered fixed and known). Speed and spacing control are applied by an active manipulation of these parameter values over time, since different sets of parameter values determine different configurations. We illustrate this in the following paragraphs. Two control laws are introduced.

In the first, the parameters $L_{i}, \omega_{i}$, and $h$ are changed abruptly, i.e., the time evolution of the parameters is represented by piecewise constant functions, exhibiting jump discontinuities at time instants $t_{k}, k=1, \ldots, M, M \in \mathbb{N}$. At every time instant $t_{k}$ the parameter values are changed and the system (7) change accordingly. The state of the new system converges to the corresponding new platoon solution, with changed velocity and/or inter-vehicle spacings. This control law is used to establish string stability properties of the system in Section IV.

In the second control law, the parameters vary continuously. New parameter values are calculated from a given new desired platoon solution, as explained in the closing paragraphs of this section. The parameter values evolve piecewise linearly in time resulting in smoother behavior of the vehicle string, as compared to the first control law. Two examples of this control law are presented at the end of this section.

The question arises how parameter values are to be chosen in order to obtain a desired platoon solution with velocity $\alpha=v_{0}$ and inter-vehicle spacings denoted by $\delta_{i}:=\beta_{i-1}-\beta_{i}>0$,

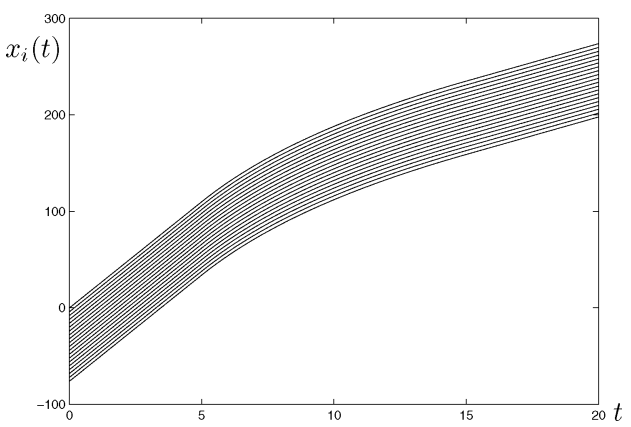

Fig. 1. Evolution of the vehicles' positions of a 39-vehicle platoon during a slowing-down maneuver with constant spacing policy. The value of $h$ varies during the time interval $[5,15] \mathrm{s}$.

$\forall i=2, \ldots, N$. For reasons of simplicity, we assume $\omega_{i}=0$. From (9b) it follows that there are $N$ parameters $L_{i}$ to determine $N-1$ constants $\delta_{i}$ :

$$
\delta_{i}=L_{i}-\frac{1}{N} \sum_{j=1}^{N} L_{j}, \quad \forall i=2, \ldots, N .
$$

One parameter, e.g., $L_{1}$, is chosen freely. Then

$$
L_{i}=\delta_{m}+L_{1}+\delta_{i}
$$

where $\delta_{m}:=\sum_{j=2}^{N} \delta_{j}$. With (14), (9a) can be rewritten as

$$
h=-\frac{L_{1}+\delta_{m}}{v_{0}}-\frac{p}{K}
$$

Notice that $L_{1}$ has to be sufficiently negative to ensure that $h>$ 0 . Equation (9) shows that the velocity of a platoon depends on $h$; inter-vehicle spacings are not influenced by this parameter. Adjusting $h$ allows to vary the velocity, while keeping intervehicle spacings constant, i.e., performing a constant spacing policy. When restricting to equal desired inter-vehicle spacings $\left(\delta_{i}=\delta, \forall i \in \tilde{N}\right):(14)$ and (15) simplify to

$$
\left\{\begin{array}{rl}
L_{i} & =N \delta+L_{1} \\
h & =-\frac{L_{1}+(N-1) \delta}{v_{0}}-\frac{p}{K}
\end{array} .\right.
$$

An example of a slowing-down maneuver with CSP in a 39-vehicle platoon is illustrated by Fig. 1. Parameter values are $p=0$, $h=1.0909, K=100, L_{1}=-100, L_{i}=-22, \forall i \neq 1$. During the time interval $[5,15] \mathrm{s}$ the value of $h$ is continuously changed into 3.0909. This results in a slowing down of the platoon from $80 \mathrm{~km} / \mathrm{h}$ to $28 \mathrm{~km} / \mathrm{h}$. The spacings stay constant at $2 \mathrm{~m}$.

A second example, displayed in Fig. 2, demonstrates the use of (16) in a speed control with constant time headway policy to slow the platoon down. Parameter values are $p=0, h=0.1$, $K=400, L_{1}=-78, L_{i}=0, \forall i \neq 1$. This corresponds to a platoon with velocity $72 \mathrm{~km} / \mathrm{h}$ and inter-vehicle spacings of $2 \mathrm{~m}$. During the time interval $[5,25] \mathrm{s}$ the values of $L_{i}, i \in \mathcal{I}$ are varied in a piecewise linear fashion. They receive the new values $L_{1}=-37.5$ and $L_{i}=0.3, \forall i \neq 1$, resulting in a platoon moving at $45 \mathrm{~km} / \mathrm{h}$ with spacings of $0.95 \mathrm{~m}$.

From (16) one concludes that each vehicle requires the values of $N, L_{1}, v_{0}$, and $\delta$ to calculate its values $L_{i}$ and $h$. This implies the need to communicate these values between the individual vehicles. Communication is only required at the time instants $t_{k}$, 


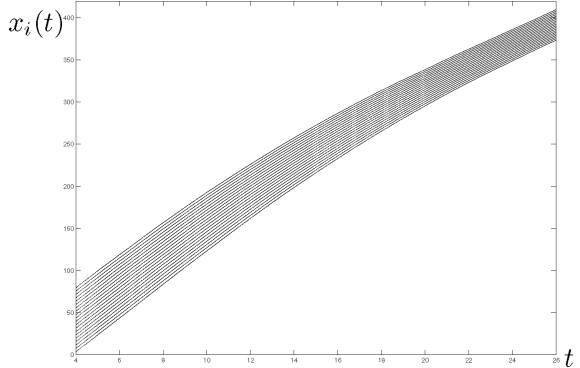

Fig. 2. Evolution of the vehicles' positions of a 39-vehicle platoon during a slowing-down maneuver with constant time headway policy.

when new desired values are created, and can be further reduced by restricting to switching between a finite number of preset platoon solutions, stored in the memory of each vehicle.

\section{Stability ANALYSIS}

In this section the stability of a platoon solution as a function of the coupling strength is investigated. Apply the following change of coordinates in (4)-(6):

$$
x_{i}(t)=\alpha t+\beta_{i}+z_{i}(t)
$$

where $\alpha$ and $\beta_{i}$ satisfy (9). Although the values $\beta_{i}$ are defined up to a constant, this does not affect the resulting equations. With $z:=\left[\begin{array}{lllll}z_{1} & \dot{z}_{1} & \cdots & z_{N} & \dot{z}_{N}\end{array}\right]^{T}$

$$
\dot{z}=A z
$$

with the system matrix $A$ identical to the system matrix of the original system (7). Since $A$ has one zero-eigenvalue, system (17) has a line of equilibrium points. This line corresponds to the platoon solution. The platoon solution is called asymptotically stable if and only if the remaining $2 N-1$ eigenvalues of $A$ are located in the open left half plane.

Theorem 2: The platoon solution (8)-(9) of system (7) is asymptotically stable if and only if

$$
K<\frac{(p+h K)^{2}}{2 \cos ^{2}\left(\frac{\pi}{N}\right)}
$$

Proof: Define $\gamma:=p+h K>0$. Since the matrix $A$ is circulant it can be block diagonalized according to (2) and (3). The matrices appearing on the diagonal are

$$
A_{i}:=\left[\begin{array}{cc}
0 & 1 \\
K\left(\omega^{(N-1)(i-1)}-1\right) & -\gamma
\end{array}\right], \quad \forall i \in \mathcal{I}
$$

with $\omega=\exp (2 \pi j / N)$. Notice that $\omega^{(N-1)(i-1)}=\omega^{1-i}$. The eigenvalues of $A_{i}$ are

$$
\lambda_{i, \pm}=-\frac{\gamma}{2} \pm \frac{1}{2}\left(\gamma^{2}+4 K\left(\omega^{(1-i)}-1\right)\right)^{1 / 2}
$$

where the square root of a complex number $a+j b$ is defined as

$$
(a+j b)^{1 / 2}:=\sqrt{\frac{|a+j b|+a}{2}}+j \operatorname{sgn}(b) \sqrt{\frac{|a+j b|-a}{2}} .
$$

The values of $K$ for which the eigenvalues belong to the open left half plane will be determined. Notice that $A_{1}$ yields the structural zero-eigenvalue and a strictly negative eigenvalue $-\gamma$. Denote the eigenvalues of $A_{i}$ as

$$
\lambda_{i, \pm}=-\frac{\gamma}{2} \pm \frac{1}{2}\left(a_{i}+j b_{i}\right)^{1 / 2}
$$

with

$$
\begin{aligned}
& a_{i}:=\gamma^{2}+4 K \cos \left(\frac{2 \pi(1-i)}{N}\right)-4 K \\
& b_{i}:=4 K \sin \left(\frac{2 \pi(1-i)}{N}\right) .
\end{aligned}
$$

We now require that

$$
-\frac{\gamma}{2} \pm \frac{1}{2} \Re e\left(a_{i}+j b_{i}\right)^{1 / 2}<0
$$

With (20), this is rewritten as

$$
-\gamma \pm \sqrt{\frac{\left|a_{i}+j b_{i}\right|+a_{i}}{2}}<0
$$

A calculation shows that this is equivalent to

$$
\begin{aligned}
K<K_{C, i} & :=\gamma^{2}\left(\frac{1-\cos \left(\frac{2 \pi(1-i)}{N}\right)}{\sin ^{2}\left(\frac{2 \pi(1-i)}{N}\right)}\right) \\
& =\frac{\gamma^{2}}{2 \cos ^{2}\left(\frac{\pi(1-i)}{N}\right) .}
\end{aligned}
$$

If and only if $K<\min _{i \in \mathcal{I} \backslash\{1\}} K_{C, i}$, the system is asymptotically stable. Since

$$
\min _{i \in \mathcal{I} \backslash\{1\}} K_{C, i}=\frac{\gamma^{2}}{2 \cos ^{2}\left(\frac{\pi}{N}\right)}
$$

the proof is concluded.

Inspection of inequality (18) yields the following corollary:

Corollary 1: Define the constants $K_{+}$and $K_{-}$by

$$
K_{ \pm}:=\frac{\left(\cos ^{2}\left(\frac{\pi}{N}\right)-p h\right) \pm \sqrt{\left(\cos ^{2}\left(\frac{\pi}{N}\right)-p h\right)^{2}-(p h)^{2}}}{h^{2}} .
$$

If the value of $h$ is such that

- $p h \geq 1 / 2 \cos ^{2}(\pi / N)$, then system (7) is asymptotically stable for all $K>0$;

- $p h<1 / 2 \cos ^{2}(\pi / N)$, then system (7) is asymptotically stable if and only if the coupling strength satisfies $0<K<$ $K_{-}$or $K>K_{+}$.

If there is a sufficient amount of friction in the system and the value of $h$ is sufficiently large, the system is asymptotically stable, independent of the value of the coupling strength. If the system is frictionless, the coupling strength receives a lower bound, the value of which depends on $h$. If in a frictionless system the control (6) does not include the velocity feedback term, i.e., $h=0$, then $K_{-}=0$ and $K_{+} \rightarrow \infty$; the system is unstable. 


\section{StRING STABILITY}

\section{A. String Stability for Ring-Interconnected Systems}

In this section, we introduce a notion of string stability adapted to the ring interconnection structure. In order to motivate our stability definition for ring coupled systems, we will present a short discussion of the classical definition. A platoon is called string stable when disturbances are attenuated as they propagate through the system [16]. When the disturbances are amplified, the system exhibits the slinky effect. Following [8], consider an infinite string of coupled systems with the following structure:

$$
\dot{x}_{i}=f\left(x_{i}, \ldots, x_{i-m}\right), \quad \forall i \in \mathbb{N}:=\{1,2, \ldots\}
$$

where $m \in \mathbb{N}, f(0, \ldots, 0)=0, x_{i} \in \mathbb{R}^{n}, x_{i}=0, \forall i \leq 0$. This system represents an infinite vehicle string if $x_{i}$ is defined as the spacing error between the $(i+1)$ th and $i$ th vehicle.

Definition 1: The origin of (25) is string stable if for any $\epsilon \in \mathbb{R}_{+}$, there exists a $\delta \in \mathbb{R}_{+}$such that

$$
\sup _{i}\left\|x_{i}(0)\right\|<\delta \Rightarrow \sup _{i}\left\|x_{i}(t)\right\|<\epsilon, \forall t \in \mathbb{R}_{+} .
$$

The definition may be applied to finite strings by truncation of the infinite string; string stability implies that the length of the finite system can be arbitrarily extended without causing an amplification of disturbance effects, see also [9].

In the present paper the interconnection structure is a ring, which cannot be treated as the truncation of an infinite ring interconnection. Hence, Definition 1 cannot be used to gain insight in system (7). A separate definition of string stability of finite systems is needed. Such a definition has been introduced in [17]. However, this definition is too weak for our purposes since it allows an increase in the norm of the states between some pairs of consecutive subsystems.

Consider a ring-interconnected system of the form

$$
\dot{x}_{i}=f_{i}\left(x_{i}, x_{i-1}, \ldots, x_{i-m}\right), \quad \forall i \in \mathcal{I}
$$

where $m<N, f_{i}(0, \ldots, 0)=0, x_{i} \in \mathbb{R}^{n}, x_{i}:=x_{N+i}, \forall i<0$.

Definition 2: System (27) is said to exhibit the slinky effect when

$$
\exists i \in \mathcal{I} \backslash\{1\}: \sup _{t \geq 0}\left\|x_{i}(t)\right\|>\sup _{t \geq 0}\left\|x_{i-1}(t)\right\| .
$$

We introduce the following definition.

Definition 3: System (27) is string stable (with respect to initial conditions) if

1) given any $\epsilon \in \mathbb{R}_{+}$, there exists a $\delta \in \mathbb{R}_{+}$such that

$$
\max _{i}\left\|x_{i}(0)\right\|<\delta \Rightarrow \max _{i}\left\|x_{i}(t)\right\|<\epsilon, \forall t \in \mathbb{R}_{+} ;
$$

2) the slinky effect is not present:

$$
\sup _{t \geq 0}\left\|x_{i}(t)\right\| \leq \sup _{t \geq 0}\left\|x_{i-1}(t)\right\|, \quad \forall i \in \mathcal{I} \backslash\{1\} .
$$

String stability with respect to initial conditions is an important property of system (7). The vehicle strings of the present paper are controlled by adapting the parameter values $L_{i}, \omega_{i}$, and $h$. Abrupt changes of these values can be modeled as a non-zero initial condition to the system. This is discussed in Section IV-C, where Definition 3 is applied to system (7).

In the next section the notion of string stability with respect to the input is introduced. An external input is added to the system, which can be interpreted as an external disturbance [18]. When this disturbance does not amplify when traveling through the string, the system is called string stable w.r.t. the input.

Remark: Most papers discussing the notion of string stability of vehicle platoons consider string stability with respect to the input. Zero initial conditions for the separation distance errors is a standard assumption. The leader vehicle has access to the input of the second vehicle [6], [19]-[21]. Its behavior is interpreted as a disturbance to the system.

\section{B. String Stability With Respect to the Input}

Let $e_{i}$ be the distance error between the $i$ th and $(i-1)$ th vehicle, with respect to the platoon solution (8)-(9)

$$
e_{i}(t):=x_{i}(t)-x_{i-1}(t)-\left(\beta_{i}-\beta_{i-1}\right)
$$

and assume the first vehicle of the platoon experiences a disturbance $u: \mathbb{R} \rightarrow \mathbb{R}$

$$
\ddot{x}_{1}+(p+h K) \dot{x}_{1}=K\left(x_{i-1}-x_{i}-L_{i}\right)+u .
$$

Notice that from (28) it follows:

$$
\sum_{j=1}^{N} \frac{\mathrm{d} e_{j}}{\mathrm{~d} t}(t)=0, \text { and } \sum_{j=1}^{N} e_{j}(t)=0, \quad \forall t \in \mathbb{R} .
$$

These equations determine a linear subspace in $\mathbb{R}^{2 N}$. With (28) and (29), the system (7) change into

$$
\begin{aligned}
\ddot{e}_{1}+(p+h K) \dot{e}_{1} & =K\left(e_{N}-e_{1}\right)+u \\
\ddot{e}_{2}+(p+h K) \dot{e}_{2} & =K\left(e_{1}-e_{2}\right)-u \\
\ddot{e}_{i}+(p+h K) \dot{e}_{i} & =K\left(e_{i-1}-e_{i}\right), \quad \forall i=3, \ldots, N .
\end{aligned}
$$

Definition 4: System (31) is string stable (with respect to the input) if

1) the system is input-to-state stable;

2) $\sup _{i} \sup _{t>0}\left\|e_{i}(t)\right\|$ is bounded when $N \rightarrow \infty$;

3) $\sup _{t \geq 0}\left\|e_{i}(t)\right\| \leq \sup _{t \geq 0}\left\|e_{i-1}(t)\right\|, \quad \forall i=3, \ldots, N$.

In the remainder of this section, string stability with respect to the input of system (31) is proven. System (31) is rewritten as a first order linear system by using $z_{i}:=\left[\begin{array}{cc}e_{i} & \dot{e}_{i}\end{array}\right]^{T}$

$$
\begin{aligned}
\dot{z}_{1}= & {\left[\begin{array}{cc}
0 & 1 \\
-K & -(p+h K)
\end{array}\right] z_{1}+\left[\begin{array}{cc}
0 & 0 \\
K & 0
\end{array}\right] z_{N}+\left[\begin{array}{l}
0 \\
1
\end{array}\right] u } \\
\dot{z}_{2}= & {\left[\begin{array}{cc}
0 & 1 \\
-K & -(p+h K)
\end{array}\right] z_{2}+\left[\begin{array}{cc}
0 & 0 \\
K & 0
\end{array}\right] z_{1}-\left[\begin{array}{l}
0 \\
1
\end{array}\right] u } \\
\dot{z}_{i}= & {\left[\begin{array}{cc}
0 & 1 \\
-K & -(p+h K)
\end{array}\right] z_{i}+\left[\begin{array}{cc}
0 & 0 \\
K & 0
\end{array}\right] z_{i-1}, } \\
& i=3, \ldots, N .
\end{aligned}
$$

The state of system (32) is confined to the linear subspace (30). The system possesses a line of equilibrium points, corresponding to the zero-eigenvalue. The origin is the only equilibrium contained in the linear subspace (30). This yields the following lemma. 
Lemma 2: If the system matrix of (32) possesses one (structural) zero-eigenvalue and all other eigenvalues have negative real part, then the origin of system (32) restricted to the hyperplane (30) is asymptotically stable.

We introduce a second lemma, presented in [22] and [23].

Lemma 3: Consider $y, h, x: \mathbb{R}_{+} \rightarrow \mathbb{R}$, with $y=h * x$ ('*' denoting the convolution operator). Denote the Laplace transform of $h$ by $H: \mathbb{C} \rightarrow \mathbb{C}$. If $|H(j \omega)| \leq 1, \forall \omega \in \mathbb{R}$ and $h$ does not change sign in $\mathbb{R}_{+}$, then $\sup _{t>0}\|y(t)\| \leq \sup _{t>0}\|x(t)\|$.

The above lemmas allow us to prove the following theorem.

Theorem 3: If $K<(p+h K)^{2} / 4$, the platoon described by (31) is string stable with respect to the input.

Proof: The system matrix of the linear system (32) is identical to the system matrix of (7). Since $K<(p+h K)^{2} / 4$, (18) holds and the system matrix has only eigenvalues with a negative real part except for one zero-eigenvalue. By Lemma 2, the system is asymptotically stable, and, consequently, input-tostate stable.

Moreover, if $K<(p+h K)^{2} / 4$, Theorem 2 implies that system (7) with $N \rightarrow \infty$ is asymptotically stable. It follows that (32) with $N \rightarrow \infty$ is asymptotically stable and input-to-state stable, yielding boundedness of $\sup _{i} \sup _{t \geq 0}\left\|e_{i}(t)\right\|$.

The structure of (31) allows the construction of the function $h: \mathbb{R} \rightarrow \mathbb{R}$ satisfying

$$
e_{i}=h * e_{i-1}, \quad i=3, \ldots, N
$$

or in the Laplace-domain

$$
E_{i}(s)=H(s) E_{i-1}(s), \quad i=3, \ldots, N .
$$

From (31) it follows that:

$$
H(s)=\frac{K}{s^{2}+(p+h K) s+K} .
$$

Notice that $|H(j \omega)| \leq 1, \forall \omega \in \mathbb{R}$ if $K<(p+h K)^{2} / 2$. Moreover, $h$ does not change sign if both poles of $H$ are real (with the extra restriction of initial conditions equal to zero); this is the case when $K<(p+h K)^{2} / 2$. Applying Lemma 3 , we conclude that $\sup _{t \geq 0}\left\|e_{i}(t)\right\| \leq \sup _{t \geq 0}\left\|e_{i-1}(t)\right\|, \forall i=$ $3, \ldots, N$ if $K<(p+h \bar{K})^{2} / 4$ holds.

Remark: Theorem 2 and Theorem 3 show that the parameters determining the stability properties of the platoon are friction $p$ and time headway $h$. In a leader-follower context it is a wellknown result that adding a velocity feedback (i.e., $h>0$ ) to each vehicle improves the string stability [23]. This agrees with the results of the present paper, where a ring interconnection is investigated.

Without this velocity feedback (i.e., $h=0$ ) a frictionless leader-follower interconnection cannot be string stable, see [6], [18]. This is also in agreement with our results.

\section{String Stability With Respect to Initial Conditions}

In the present section string stability is investigated with respect to a particular class of initial conditions. Section II-C showed how one steers the system behavior from one platoon solution to another, by adjusting the parameters $L_{i}, \omega_{i}$, and $h$. Assume these parameter values are changed (abruptly) at some time $t_{k}$. Since, according to (9), $\beta_{i}$ depends on $L_{i}$ and $\omega_{i}$, the change of coordinates (28) is altered. A new set of spacing

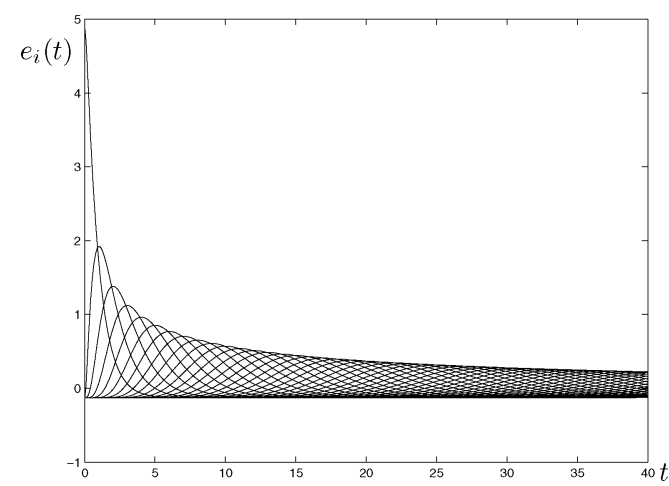

Fig. 3. Evolution of the separation distance errors for a platoon of 39 vehicles.

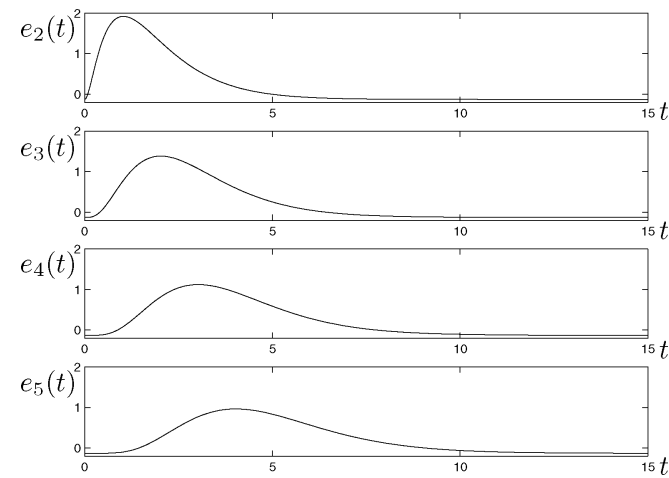

Fig. 4. Separation distance errors of the first five vehicles.

errors $e_{i}$ describes the state of the platoon. The initial value of these new spacing errors is the difference of the spacings $\delta_{i}$ before and after $t_{k}$.

In the remainder of this section, string stability with respect to a slowing-down maneuver is examined. Similar behavior is obtained for speeding-up maneuvers. For simplicity, $\omega_{i}=0$, $\forall i \in \mathcal{I}$ is assumed. Assume that for time $t<0$ the system is at the platoon solution. At $t=0$ the set point $L_{1}$ is replaced by $\tilde{L}_{1}$. The corresponding initial condition is given by

$$
\left\{\begin{array}{l}
e_{1}(0)=\frac{N-1}{N}\left(\tilde{L}_{1}-L_{1}\right) \\
e_{i}(0)=\frac{L_{1}-\tilde{L}_{1}}{N}, \quad \forall i \in \mathcal{I} \backslash\{1\} \\
\dot{e}_{i}(0)=0, \forall i \in \mathcal{I} .
\end{array}\right.
$$

In Fig. 3, system (31) is simulated with $N=39, \tilde{L}_{1}=L_{1}+5$, $p=0, h=1, K=10$. The corresponding initial conditions are $e_{1}(0)=4.8718, e_{i}(0)=-0.1282, \forall i \neq 1$ and $\dot{e}_{i}(0)=0$, $\forall i \in \mathcal{I}$.

Fig. 3 presents the evolution of the distance errors over time. For reasons of clarity, the distance errors with even index are omitted from the picture. The figure suggests that the maximum distance error between pairs of consecutive vehicles does not grow when proceeding towards the tail of the platoon. This is made explicit by the separate plots of Fig. 4, where the first four separation distance errors are displayed.

The solid line in Fig. 5 represents the distance error $e_{4}$ over a longer time period and illustrates a typical feature of the interconnection topology: each distance error rises quickly to its maximum value and then decreases to a value close to zero, but, contrary to leader follower control, it keeps oscillating with decreasing peaks over longer periods. The simulations show that 


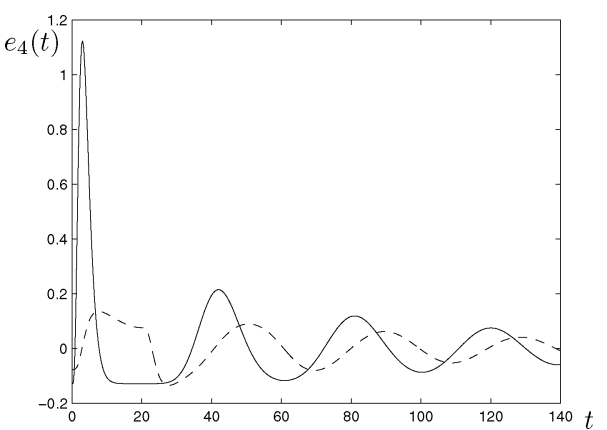

Fig. 5. Separation distance error $e_{4}(t)$. The solid line corresponds to an abrupt change of parameter value $L_{1}$ at $t=0$. The dashed line represents the error when $L_{1}$ is linearly increased in the time interval $[0,20]$.

the interconnection topology of the present paper is string stable w.r.t. initial conditions.

It is not possible to completely remove the oscillations. However, the amplitude of the oscillations can be significantly reduced, as illustrated by the dashed curve in Fig. 5. This curve is produced by applying a linear adaption in time as explained in Section II-C. The parameter value of $L_{1}$ increases linearly to the value $L_{1}(0)+5$ during an interval of $20 \mathrm{~s}$. Compared to the maneuver where the value of $L_{1}$ was changed abruptly, the behavior is significantly improved: the sharp peak at $t=4$ is removed and the oscillations are smaller in amplitude.

\section{ROBUSTNESS}

In this section some robustness properties of system (7) are discussed, by means of an example. The initial state of a string of 39 vehicles is given by $\dot{x}_{i}(0)=0, x_{i}(0)=1-i, \forall i=1, \ldots, 39$. Friction is absent $(p=0)$, and $h=0.06, K=800$. The set points are chosen $L_{1}=-70, L_{i}=1, \forall i \neq 1, \omega_{i}=0$, $\forall i \in \mathcal{I}$. The vehicle string forms a platoon moving at $49 \mathrm{~km} / \mathrm{h}$. Assume that one of the vehicles starts to malfunction; its velocity is bounded from above by $5.4 \mathrm{~km} / \mathrm{h}$. The vehicle can no longer attain the velocity of $49 \mathrm{~km} / \mathrm{h}$ required by the platoon. In the case of leader-follower control this would cause the leading group of vehicles to abandon the group with the malfunctioning vehicle as first vehicle, and therefore a splitting of the platoon. The distance between both groups would increase without bound. With the interconnection topology of the present paper all vehicles adapt to the "weakest link" and the platoon sets off at the maximum velocity feasible by the malfunctioning vehicle. This is illustrated in Fig. 6: at $t=50$ s the speed of one of the vehicles becomes bounded by $5.4 \mathrm{~km} / \mathrm{h}$. The distance between the first and the second group remains bounded. There is a splitting of the platoon but no abandoning. For reasons of clarity of the picture, only the positions of the vehicles with an odd index are plotted.

\section{CONCLUSION}

In this paper, a novel strategy for longitudinal control of vehicle strings is proposed. The vehicles are interconnected at the interaction level into a unidirectional ring; no leader/master vehicle is present. Each vehicle measures the distance with its immediate forward neighbor, and the lead vehicle in the platoon receives information on the position of the last vehicle, thus establishing a ring structure on the control level. We prove that the

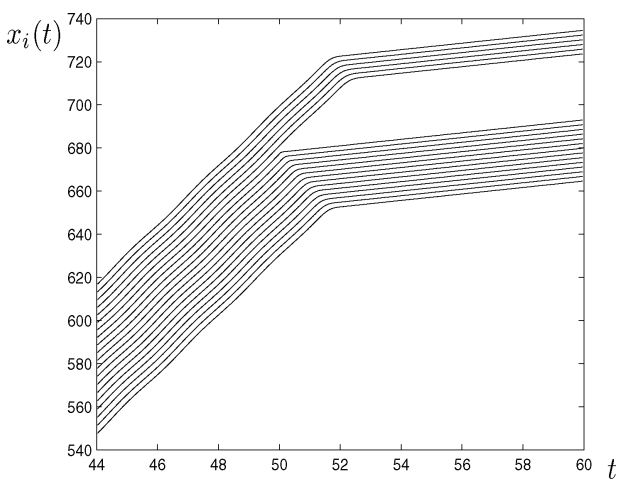

Fig. 6. Evolution of the position for a platoon of 39 vehicles. At $t=50 \mathrm{~s}$, the 12 th vehicle starts malfunctioning and cannot drive faster than $5.4 \mathrm{~km} / \mathrm{h}$.

resulting behavior of the system is a platoon of vehicles moving at a constant velocity with constant inter-vehicle spacings.

We construct algorithms to control the platoon behavior. By adjusting parameter values in the system, the platoon speeds up or slows down, while inter-vehicle spacings are regulated to desired values. The concept of string stability of a platoon is discussed and applied to the proposed control algorithms, showing that the ring interconnection behaves as desired with respect to string stability.

The paper is concluded by a demonstration of a typical robustness property. When one of the vehicles malfunctions, i.e., its speed is bounded from above, the platoon does not break in two parts.

\section{ACKNOWLEDGMENT}

The authors would like to thank the anonymous referees for their useful comments and questions.

\section{REFERENCES}

[1] W. S. Levine and M. Athans, "On the optimal error regulation of a string of moving vehicles," IEEE Trans. Automat. Control, vol. AC-11, no. 3, pp. 355-361, Mar. 1966.

[2] S. M. Melzer and B. C. Kuo, "Optimal regulation of systems described by a countably infinite number of objects," Automatica, vol. 7, pp. 359-366, 1971.

[3] J. L. Willems, "Optimal control of a uniform string of moving vehicles," Ricerche di Automatica, vol. 2, no. 2, pp. 184-192, 1971.

[4] M. R. Jovanović and B. Bamieh, "On the ill-posedness of certain vehicular platoon control problems," IEEE Trans. Automat. Control, vol. 50, no. 9, pp. 1307-1321, Sep. 2005.

[5] S. S. Stanković, M. J. Stanojević, and D.Šiljak, "Decentralized overlapping control of a platoon of vehicles," IEEE Trans. Control. Syst. Technol., vol. 8, no. 5, pp. 816-832, Sep. 2000.

[6] P. A. Ioannou and C. C. Chien, "Autonomous intelligent cruise control," IEEE Trans. Veh. Technol., vol. 42, no. 4, pp. 657-672, 1993.

[7] Z. Jin and R. M. Murray, "Double-graph control strategy of multi-vehicle formations," in Proc. 43rd IEEE Conf. Dec. Control, 2004, pp. 1988-1994.

[8] D. Swaroop and J. K. Hedrick, "String stability of interconnected systems," IEEE Trans. Automat. Control, vol. 41, no. 3, pp. 349-356, Mar. 1996.

[9] P. A. Cook, "Conditions for string stability," Syst. Control Lett., vol. 54, no. 10, pp. 991-998, 2005.

[10] Z. Lin, M. Broucke, and B. Francis, "Local control strategies for groups of mobile autonomous agents," IEEE Trans. Automat. Control, vol. 49, no. 4, pp. 622-629, Apr. 2004.

[11] J. A. Marshall, M. E. Broucke, and B. A. Francis, "Formations of vehicles in cyclic pursuit," IEEE Trans. Automat. Control, vol. 49, no. 11, pp. 1963-1974, Nov. 2004.

[12] S. L. Smith, M. E. Broucke, and B. A. Francis, "A hierarchical cyclic pursuit scheme for vehicle networks," Automatica, vol. 41, no. 6, pp. 1045-1053, 2005. 
[13] J. A. Rogge and D. Aeyels, "Stability of phase locking in a ring of unidirectionally coupled oscillators," J. Phys. A, vol. 37, pp. 11135-11148, 2004.

[14] P. J. Davis, Circulant Matrices. London, U.K.: Chelsea, 1979.

[15] B. Bamieh, F. Paganini, and M. Dahleh, "Distributed control of spatially invariant systems," IEEE Trans. Automat. Control, vol. 47, no. 7, pp. 1091-1107, Jul. 2002.

[16] L. E. Peppard, "String stability of relative-motion pid vehicle control systems," IEEE Trans. Automat. Control, vol. AC-19, no. 10, pp. 579-581, Oct. 1974.

[17] A. Pant, P. Seiler, and K. Hedrick, "Mesh stability of look-ahead interconnected systems," IEEE Trans. Automat. Control, vol. 47, no. 2, pp. 403-407, Feb. 2002.

[18] R. J. Caudill and W. L. Garrard, "Vehicle-follower longitudinal control for automated transit vehicles," J Dyn. Syst.-T. ASME, pp. 241-248, 1977.

[19] C.-Y. Liang and H. Peng, "Optimal adaptive cruise control with guaranteed string stability," Veh. Syst. Dyn., vol. 31, pp. 313-330, 1999.

[20] D. Swaroop, J. K. Hedrick, C. C. Chien, and P. Ioannou, "A comparison of spacing and headway control laws for automatically controlled vehicles," Veh. Syst. Dyn., vol. 23, pp. 597-625, 1994.

[21] D. Yanakiev and I. Kanellakopoulos, "Nonlinear spacing policies for automated heavy-duty vehicles," IEEE Trans. Veh. Technol., vol. 47, no. 4, pp. 1365-1377, Nov. 1998.

[22] J. K. Hedrick and D. Swaroop, "Dynamic coupling in vehicles under automatic control," Veh. Syst. Dyn., vol. 23, pp. 209-217, 1994

[23] J. Eyre, D. Yanakiev, and L. Kanellakopoulos, "A simplified framework for string stability analysis of automated vehicles," Veh. Syst. Dyn., vol. 30, no. 5, pp. 375-405, 1998.

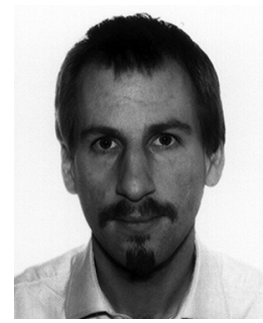

Jonathan A. Rogge received the M.S. degree in physics engineering and the Ph.D. degree from Ghent University, Ghent, Belgium, in 2000 and 2006, respectively.

After receiving the Ph.D. degree he held a postdoctoral appointment for three months with the Department of Control Methods, Technical University of Bratislava, Slovakia. He is currently working in the SYSTeMS Group, Ghent University. His current research interests include interconnections of oscillating systems, systems consisting of strings of vehicles, and multiple mobile robot systems.

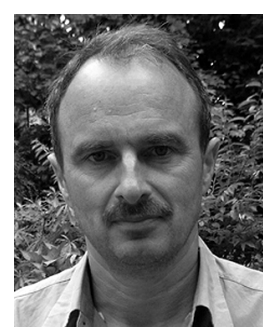

Dirk Aeyels received the B.S. degree in electrical and mechanical engineering from the Universiteit Gent, Ghent, Belgium, and the M.Sc. and D.Sc. degrees in systems science and mathematics from Washington University, St. Louis, MO

He has been a Visiting Professor at the Université Catholique de Louvain, Belgium. At present he is a Full Professor in Networks of Nonlinear Systems, Control and Optimization at the Faculty of Engineering at the Universiteit Gent, and Director of The SYSTeMS Research Group. He has been Associate Editor of Systems \& Control Letters, the Siam Journal on Control and Optimization (SICON), and the European Journal of Control. Current fields of interest include networks of nonlinear systems, multirobot systems, neural networks, and economical systems. 